Supporting information for:

\title{
Application of chemometrics for coal pyrolysis products by online py-GC×GC-MS
}

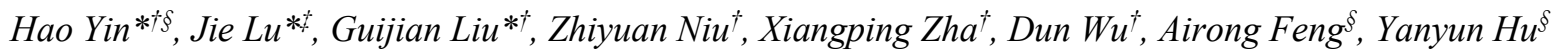

${ }^{\dagger}$ CAS Key Laboratory of Crust-Mantle Materials and the Environments, School of Earth and Space Sciences, University of Science and Technology of China, Hefei 230026, China.

†National High Magnetic Field Laboratory, Florida State University, 1800 East Paul Dirac Drive, Tallahassee, Florida 32310-4005, United States.

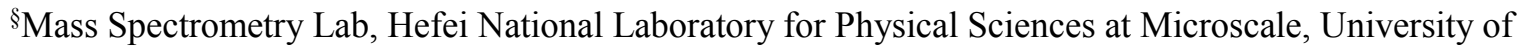
Science and Technology of China, Hefei 230026, China.

\section{Contents:}

The detailed hydropyrolysis conditions for XQ8 and XQ13_1 investigated by Wang et al. with the coal tar yields are shown. 
Both of XQ8 and XQ13_1 from Xieqiao coalmine were performed to hydropyrolysis under three conditions by Wang, J.-R., etc. (Study on direct liquefaction character of Huainan gas coal. Clean Coal Technol. 2001). The first condition are the reaction temperate of $450{ }^{\circ} \mathrm{C}$ and $10 \mathrm{MPa}$ of $\mathrm{H}_{2}$ pressure with the reaction time of 60 minutes. The second condition are the reaction temperature of $460{ }^{\circ} \mathrm{C}$ and $10 \mathrm{MPa}$ of $\mathrm{H}_{2}$ pressure with the reaction time of 60 minutes. And the third condition are reaction temperature of $460{ }^{\circ} \mathrm{C}$ and $10 \mathrm{MPa}$ of $\mathrm{H}_{2}$ pressure with the reaction time of 120 minutes. Under the first condition, the coal tar of XQ8 is 52.55\% and XQ13_1 is 49.84\%. Under the second condition, the coal tar of XQ8 is $57.90 \%$ and XQ13_1 is $58.78 \%$. Under the third condition, the coal tar of XQ8 is $61.69 \%$ and XQ13_1 is $62.09 \%$. 